\title{
APPROXIMATING CRITICAL PARAMETERS OF BRANCHING RANDOM WALKS
}

\author{
DANIELA BERTACCHI, ${ }^{*}$ Università di Milano - Bicocca \\ FABIO ZUCCA, ${ }^{* *}$ Politecnico di Milano
}

\begin{abstract}
Given a branching random walk on a graph, we consider two kinds of truncations: either by inhibiting the reproduction outside a subset of vertices or by allowing at most $m$ particles per vertex. We investigate the convergence of weak and strong critical parameters of these truncated branching random walks to the analogous parameters of the original branching random walk. As a corollary, we apply our results to the study of the strong critical parameter of a branching random walk restricted to the cluster of a Bernoulli bond percolation.
\end{abstract}

Keywords: Branching random walk; critical parameters; percolation; graphs

2000 Mathematics Subject Classification: Primary 60K35

\section{Introduction}

The branching random walk is a process which serves as a (rough) model for a population living in a spatially structured environment (the vertices of a-possibly oriented-graph $(X, \mathcal{E}(X)))$, where each individual lives in a vertex, breeds, and dies at random times, and each offspring is placed (randomly) in one of the neighboring vertices. There is no bound on the number of individuals allowed per site. The vertices may be thought of as small ecosystems or squares of soil (with their proximity connections - the edges) and individuals as animals or plants. Depending on the parameters involved and on the nature of $(X, \mathcal{E}(X))$, the population may face almost-sure extinction, global survival (i.e. with positive probability, at any time there will be at least one individual alive), or local survival (i.e. with positive probability, at arbitrarily large times there will be at least one individual alive in a fixed vertex). These matters have been investigated by several authors (see [11], [12], [13], [16], [19], and [23], among others, and [15] for more references).

Let us be more precise as to the definition of the process and of the environment. The graph $(X, \mathcal{E}(X))$ is endowed with a weight function $\mu: X \times X \rightarrow[0,+\infty)$ such that $\mu(x, y)>0$ if and only if $(x, y) \in \mathcal{E}(X)$ (in which case we write $x \rightarrow y$ ). We call the couple $(X, \mu) \mathrm{a}$ weighted graph. We require that there exists $K<+\infty$ such that $k(x):=\sum_{y \in X} \mu(x, y) \leq K$ for all $x \in X$ (other conditions will be stated in Section 2).

Given $\lambda>0$, the branching random walk $(\mathrm{BRW}(X)$ or, for short, BRW) is the continuoustime Markov process $\left\{\eta_{t}\right\}_{t \geq 0}$, with configuration space $\mathbb{N}^{X}$, where each existing particle at $x$ has an exponential lifespan of parameter 1 and, during its life, breeds at the arrival times of a

Received 15 August 2008; revision received 30 March 2009.

* Postal address: Dipartimento di Matematica e Applicazioni, Università di Milano - Bicocca, Via Cozzi 53, 20125 Milano, Italy. Email address: daniela.bertacchi@unimib.it

** Postal address: Dipartimento di Matematica, Politecnico di Milano, Piazza Leonardo da Vinci 32, 20133 Milano, Italy. Email address: fabio.zucca@polimi.it 
Poisson process of parameter $\lambda k(x)$ and then chooses to send its offspring to $y$ with probability $\mu(x, y) / k(x)$ (note that $(\mu(x, y) / k(x))_{x, y \in X}$ is the transition matrix of a random walk on $X$ ). In the literature we usually find the particular case $k(x)=1$ for all $x \in X$ (i.e. the breeding rate is constant among locations - no place is more fertile than others) or, sometimes, the case where $\mu=\mathbf{1}_{\varepsilon(X)}$ (i.e. the breeding rate is proportional to the degree and all edges have the same rate).

Two critical parameters are associated to the BRW: the weak (or global) survival critical parameter $\lambda_{\mathrm{w}}$ and the strong (or local) survival critical parameter $\lambda_{\mathrm{s}}$. They are defined as

$$
\begin{aligned}
\lambda_{\mathrm{w}} & :=\inf \left\{\lambda>0: \mathrm{P}^{\delta_{x_{0}}}\left(\text { there exists } t: \eta_{t}=\underline{0}\right)<1\right\}, \\
\lambda_{\mathrm{s}} & :=\inf \left\{\lambda>0: \mathrm{P}^{\delta_{x_{0}}}\left(\text { there exists } \bar{t}: \eta_{t}\left(x_{0}\right)=0 \text { for all } t \geq \bar{t}\right)<1\right\},
\end{aligned}
$$

where $x_{0}$ is a fixed vertex, $\underline{0}$ is the configuration with no particles at all sites, and $\mathrm{P}^{\delta_{x_{0}}}$ is the law of the process which starts with one individual in $x_{0}$. Note that if the weighted graph is connected then these values do not depend on the initial configuration, provided that this configuration is finite (that is, it has only a finite number of individuals), or on the choice of $x_{0}$. See Section 2 for a discussion on the values of $\lambda_{w}$ and $\lambda_{s}$.

When $(X, \mu)$ is infinite (and connected), the BRW is, so to speak, unbounded in two respects: the environment, since individuals may live at arbitrarily large distances from their ancestors (actually, $n$th generation individuals may live at distance $n$ from their ancestor), and the colonies' size, since an arbitrarily large number of individuals may pile up on any vertex. Hence, it is natural to consider 'truncated' BRWs where either space or colonies are bounded, and investigate the relationship between these processes and the BRW. Indeed, in the literature we often find problems tackled first in finite or compact spaces and then reached through a 'thermodynamical limit' procedure. We can see easily that it is possible to construct the BRW either from the process on finite sets (spatial truncation) or from the process on infinite space and a bound on the number of particles per site (particles truncation). In both cases the truncated process, for any fixed time $t$, converges almost surely to the BRW.

First, we consider 'spatially truncated' BRWs. We choose a family of weighted subgraphs $\left\{\left(X_{n}, \mu_{n}\right)\right\}_{n \in \mathbb{N}}$ such that $X_{n} \uparrow X, \mu_{n}(x, y) \leq \mu(x, y)$, and $\mu_{n}(x, y) \rightarrow \mu(x, y)$ as $n \rightarrow \infty$ for all $x$ and $y$. The process $\operatorname{BRW}\left(X_{n}\right)$ can be seen as the $\operatorname{BRW}(X)$ with the constraint that reproductions outside $X_{n}$ are deleted and the ones from $x$ to $y\left(x, y\right.$ in $\left.X_{n}\right)$ are removed with probability $1-\mu_{n}(x, y) / \mu(x, y)$. It is not difficult to see that, for any fixed $t$, as $n$ goes to $\infty$, the BRW $\left(X_{n}\right)$ converges to the BRW almost surely. Our first result is that $\lambda_{\mathrm{s}}\left(X_{n}\right) \rightarrow \lambda_{\mathrm{s}}(X)$ as $n \rightarrow \infty$ (where $\lambda_{\mathrm{s}}(X)$ is the strong survival critical parameter of the BRW on $(X, \mu)$ ). Indeed, we prove a slightly more general result (see Theorem 3.1) which allows us to show that if $X=\mathbb{Z}^{d}$ and $X_{n}$ is the infinite cluster of the Bernoulli bond percolation of parameter $p_{n}$, where $p_{n} \rightarrow 1$ as $n \rightarrow \infty$ sufficiently fast, then $\lambda_{\mathrm{S}}\left(X_{n}\right) \rightarrow \lambda_{\mathrm{s}}(X)$ as $n \rightarrow \infty$ almost surely with respect to the percolation probability space (see Section 7).

Second, we consider BRWs where at most $m$ individuals per site are allowed (thus taking values in $\left.\{0,1, \ldots, m\}^{X}\right)$. We call this process $\mathrm{BRW}_{m}$ and denote it by $\left\{\eta_{t}^{m}\right\}_{t \geq 0}$. Note that if $m=1$, we obtain the contact process (indeed, the $\mathrm{BRW}_{m}$ is sometimes referred to as a 'multitype contact process'-see, for instance, [18]). It is easily seen that, for all fixed $t$, we have $\eta_{t}^{m} \rightarrow \eta_{t}$ as $m \rightarrow \infty$ almost surely (see, for instance, [19], where the authors suggested this limit as a way to construct the BRW). Clearly, for all $m \geq 1$, we may consider the critical parameters $\lambda_{\mathrm{w}}^{m}$ and $\lambda_{\mathrm{s}}^{m}$ defined as in (1.1) with $\eta_{t}^{m}$ in place of $\eta_{t}$. One of the main questions we investigate in this paper is whether $\lambda_{\mathrm{w}}^{m} \rightarrow \lambda_{\mathrm{w}}$ as $m \rightarrow \infty$ and $\lambda_{\mathrm{s}}^{m} \rightarrow \lambda_{\mathrm{s}}$ as $m \rightarrow \infty$ : to the 
authors' knowledge, this is still unknown even for the case where $X=\mathbb{Z}^{d}$ with $\mu$ a transition matrix of the simple random walk.

Here is a brief outline of the paper. In Section 2 we state the basic terminology and assumptions needed in the sequel. Section 3 is devoted to the spatial approximation of the strong critical parameter $\lambda_{\mathrm{s}}$ by finite or infinite sets (see Theorems 3.1 and 3.1, respectively). We note that results on the spatial approximation, in the special case where $X=\mathbb{Z}^{d}$ and $\mu$ is the transition matrix of the simple random walk, were obtained in [17] using a different approach. In Section 4 we introduce the technique we use to prove convergence of the critical parameters of the $\mathrm{BRW}_{m}$. The technique is essentially a suitable coupling with a supercritical oriented bond percolation: this kind of comparison was introduced in [4], discussed in [6], and later applied in several papers (see, for instance, [7], [21], [22], and [24]). Nevertheless, the coupling here is quite tricky; therefore, we describe it in four steps which can be adapted to different graphs. In Section 5 we prove that $\lambda_{\mathrm{s}}^{m}$ converges to $\lambda_{\mathrm{s}}$ under some assumptions on the graph (see Theorem 5.1). As a corollary, we have $\lambda_{\mathrm{s}}^{m} \rightarrow \lambda_{\mathrm{s}}$ as $m \rightarrow \infty$ for $\mathbb{Z}^{d}$ with the simple random walk. The same approach is used in Section 6 to prove the convergence of the sequence $\lambda_{\mathrm{w}}^{m}$ to $\lambda_{\mathrm{w}}$ when $X=\mathbb{Z}^{d}$ (see Theorem 6.1 and Corollary 6.1, and Remark 6.1 for a slightly more general class of graphs) or when $X$ is a homogeneous tree (see Theorem 6.2). The results of Section 3 are applied in Section 7 in order to study the strong critical parameter of a BRW restricted to a random subgraph generated by a Bernoulli bond percolation process. Section 8 is devoted to final remarks and open questions.

\section{Terminology and assumptions}

In this section we state our assumptions on the graph $(X, \mu)$; we also recall the description of the BRW through its generator and the associated semigroup, and discuss the values of $\lambda_{\mathrm{w}}$ and $\lambda_{\mathrm{s}}$.

Given the (weighted) graph $(X, \mu)$, the degree of a vertex $x, \operatorname{deg}(x)$ is the cardinality of the set $\{y \in X: x \rightarrow y\}$; we require that $(X, \mu)$ is with bounded geometry, that is, $\sup _{x \in X} \operatorname{deg}(x)<$ $+\infty$. Moreover, we consider $(X, \mu)$ connected, which, by our definition of $\mu$ (recall that $\mu(x, y)>0$ if and only if $(x, y) \in \mathcal{E}(X))$, is equivalent to $\mu^{(n)}(x, y)>0$ for some $n=n(x, y)$, where $\mu^{(n)}$ is the $n$th power of the matrix $\mu$. When $(\mu(x, y))_{x, y}$ is stochastic (i.e. $k(x)=1$ for all $x \in X)$, in order to stress this property we use the notation $P, p(x, y)$, and $p^{(n)}(x, y)$ instead of $\mu, \mu(x, y)$, and $\mu^{(n)}(x, y)$. Define

$$
d(x, y)=\min \left\{n: \text { there exists }\left\{x_{i}\right\}_{i=0}^{n}, x_{0}=x, x_{n}=y, x_{i} \rightarrow x_{i+1}\right\} ;
$$

note that this is a true metric on $X$ if and only if $(X, \mu)$ is nonoriented.

We need to define the product of two graphs (in our paper these will be space/time products): given two graphs $(X, \mathcal{E}(X))$ and $(Y, \mathcal{E}(Y))$, we denote by $(X, \mathcal{E}(X)) \times(Y, \mathcal{E}(Y))$ the weighted graph with the set of vertices $X \times Y$ and the set of edges $\mathcal{E}=\left\{\left((x, y),\left(x_{1}, y_{1}\right)\right):\left(x, x_{1}\right) \in\right.$ $\left.\mathcal{E}(X),\left(y, y_{1}\right) \in \mathcal{E}(Y)\right\}$ (in Figure 1 (a) we draw the connected component of $\mathbb{Z} \times \mathbb{Z}$ containing $(0,0))$. Besides, by $(X, \mathcal{E}(X)) \square(Y, \mathscr{E}(Y))$ we mean the graph with the same vertex set as before and vertices $\mathcal{E}=\left\{\left((x, y),\left(x_{1}, y_{1}\right)\right):\left(x, x_{1}\right) \in \mathcal{E}(X), y=y_{1}\right\} \cup\left\{\left((x, y),\left(x_{1}, y_{1}\right)\right): x=\right.$ $\left.x_{1},\left(y, y_{1}\right) \in \mathcal{E}(Y)\right\}$ (see Figure 1(b)).

Let $\left\{\eta_{t}\right\}_{t \geq 0}$ be the branching random walk on $X$ with parameter $\lambda$, associated to the weight function $\mu$ : the configuration space is $\mathbb{N}^{X}$ and its generator is

$$
\mathcal{L} f(\eta):=\sum_{x \in X} \eta(x)\left(\partial_{x}^{-} f(\eta)+\lambda \sum_{y \in X} \mu(x, y) \partial_{y}^{+} f(\eta)\right),
$$




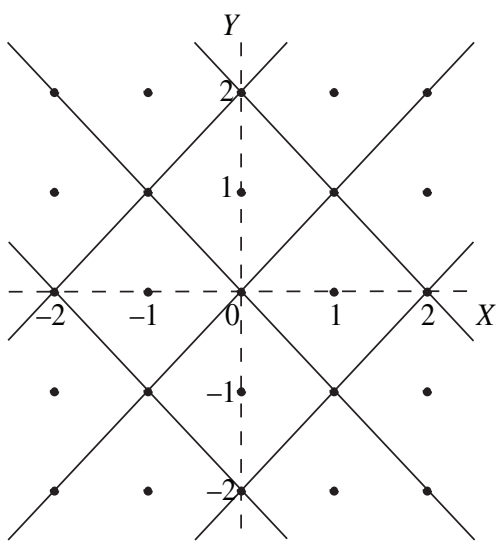

(a)

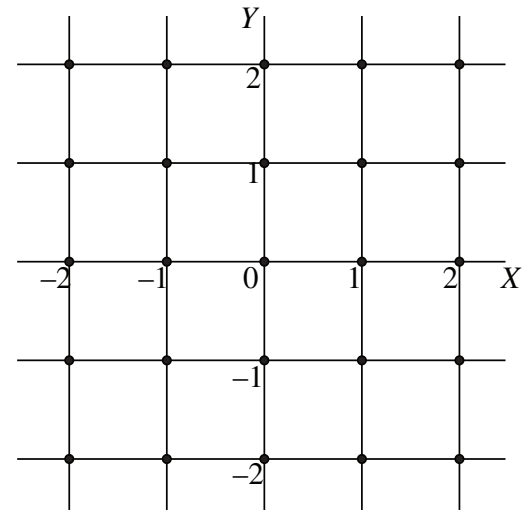

(b)

Figure 1: (a) $X \times Y(X=Y=\mathbb{Z})$. (b) $X \square Y(X=Y=\mathbb{Z})$.

where $\partial_{x}^{ \pm} f(\eta):=f\left(\eta \pm \delta_{x}\right)-f(\eta)$. Analogously, the generator of the $\mathrm{BRW}_{m}\left\{\eta_{t}^{m}\right\}_{t \geq 0}$ is

$$
\mathcal{L}_{m} f(\eta):=\sum_{x \in X} \eta(x)\left(\partial_{x}^{-} f(\eta)+\lambda \sum_{y \in X} \mu(x, y) \mathbf{1}_{[0, m-1]}(\eta(y)) \partial_{y}^{+} f(\eta)\right) .
$$

Note that the configuration space is still $\mathbb{N}^{X}$ (though we may consider $\{0,1, \ldots, m\}^{X}$ as well). The semigroup $S_{t}$ is defined as $S_{t} f(\eta):=\mathrm{E}^{\eta}\left(f\left(\eta_{t}\right)\right)$, where $f$ is any function on $\mathbb{N}^{X}$ such that the expected value is defined.

The strong and weak survival critical parameters of the BRW clearly depend on the weighted graph $(X, \mu)$; we denote them by $\lambda_{\mathrm{S}}(X, \mu)$ and $\lambda_{\mathrm{W}}(X, \mu)$ (or simply by $\lambda_{\mathrm{s}}(X)$ and $\lambda_{\mathrm{W}}(X)$ or $\lambda_{\mathrm{s}}$ and $\lambda_{\mathrm{w}}$ ). Analogously, we denote by $\lambda_{\mathrm{s}}^{m}(X, \mu)$ and $\lambda_{\mathrm{w}}^{m}(X, \mu)$ (or simply by $\lambda_{\mathrm{s}}^{m}(X)$ and $\lambda_{\mathrm{w}}^{m}(X)$ or $\lambda_{\mathrm{s}}^{m}$ and $\left.\lambda_{\mathrm{w}}^{m}\right)$ the critical parameters of the $\mathrm{BRW}_{m}$ on $(X, \mu)$. It is known (see, for instance, [1] and [2]) that $\lambda_{\mathrm{s}}=R_{\mu}:=1 / \lim \sup _{n} \sqrt[n]{\mu^{(n)}(x, y)}$ (which is easily seen to be independent of $x, y \in X$ since the graph is connected). On the other hand, the explicit value of $\lambda_{\mathrm{w}}$ is not known in general. Nevertheless, in many cases it is possible to prove that $\lambda_{\mathrm{w}}=1 / \lim \sup _{n} \sqrt[n]{\sum_{y \in X} \mu^{(n)}(x, y)}$ (see [1] and [2]). In particular, if $k(x)=K$ for all $x \in X$ then $\lambda_{\mathrm{w}}=1 / K$; thus, if $(\mu(x, y))_{x, y}$ is a stochastic matrix then $\lambda_{\mathrm{w}}=1$.

The two critical parameters coincide (i.e. there is no pure weak phase) in many cases: if $X$ is finite or, when $\mu=P$ is stochastic, if $R=1$. Here are two sufficient conditions for $R=1$ (when $\mu=P$ is stochastic).

1. $(X, P)$ is the simple random walk on a nonoriented graph, and the ball of radius $n$ and center $x$ has subexponential growth $\left(\sqrt[n]{\left|B_{n}(x)\right|} \rightarrow 1\right.$ as $\left.n \rightarrow \infty\right)$. Indeed, for any reversible random walk, the following universal lower bound holds:

$$
p^{(2 n)}(x, x) \geq \frac{v(x)}{v\left(B_{n}(x)\right)}
$$

(see [5, Lemma 6.2]), where $v$ is a reversibility measure. If $P$ is the simple random walk then $v$ is the counting measure and the claim follows. 
An explicit example is the simple random walk on $\mathbb{Z}^{d}$ or on $d$-dimensional combs (see [25, Section 2.21] for the definition of a comb).

2. $(X, P)$ is a symmetric, irreducible random walk on an amenable group (see [25, Section 12.A]).

\section{Spatial approximation}

In this section we consider spatial truncations of the BRW. In Lemma 3.1 and Theorem 3.1, below, $\left\{X_{n}\right\}_{n \in \mathbb{N}}$ will be a sequence of finite subsets of $X$ such that $X_{n} \subseteq X_{n+1}$ and $\bigcup_{n=1}^{\infty} X_{n}=X$; we denote by ${ }_{n} \mu$ the truncation matrix defined by ${ }_{n} \mu:=\mu_{\mid X_{n} \times X_{n}}$. We define $_{n} R_{\mu}:=1 / \lim \sup _{k \rightarrow \infty} \sqrt[k]{n \mu^{(k)}(x, y)}$.

Lemma 3.1. Let $\left\{X_{n}\right\}_{n \in \mathbb{N}}$ be such that $\left(X_{n}, n_{n} \mu\right)$ is connected for all $n$. Then ${ }_{n} R_{\mu} \geq_{n+1} R_{\mu}$ for all $n$ and, when $X_{n} \subsetneq X_{n+1}$, we have ${ }_{n} R_{\mu}>_{n+1} R_{\mu}$. Moreover, ${ }_{n} R_{\mu} \downarrow R_{\mu}$.

Proof. This is essentially Theorem 6.8 of [20].

The next result is a generalization of this lemma and it goes beyond the pure spatial approximation by finite subsets. We omit the proof which follows easily from Lemma 3.1.

Theorem 3.1. Let $\left\{\left(Y_{n}, \mu_{n}\right)\right\}_{n \in \mathbb{N}}$ be a sequence of connected weighted graphs, and let $\left\{X_{n}\right\}_{n \in \mathbb{N}}$ be such that $Y_{n} \supseteq X_{n}$. Let us suppose that $\mu_{n}(x, y) \leq \mu(x, y)$ for all $n \in \mathbb{N}, x, y \in Y_{n}$, and $\mu_{n}(x, y) \rightarrow \mu(x, y)$ for all $x, y \in X$. If $\left(X_{n},{ }_{n} \mu\right)$ is connected for every $n \in \mathbb{N}$ then $\lambda_{\mathrm{s}}\left(Y_{n}, \mu_{n}\right) \geq \lambda_{\mathrm{s}}(X, \mu)$ and $\lambda_{\mathrm{s}}\left(Y_{n}, \mu_{n}\right) \rightarrow \lambda_{\mathrm{s}}(X, \mu)$ as $n \rightarrow \infty$.

A simple situation where the previous theorem applies is the nonoriented case $(\mu(x, y)>0$ if and only if $\mu(y, x)>0)$, where $X_{n}=Y_{n}$ is the ball of radius $n$ with center at a fixed vertex $x_{0}$ of $X$.

Remark 3.1. If $Y_{n}$ is finite for all $n$ then $\lambda_{\mathrm{W}}\left(Y_{n}\right)=\lambda_{\mathrm{s}}\left(Y_{n}\right)$; hence, $\lambda_{\mathrm{W}}\left(Y_{n}\right) \rightarrow \lambda_{\mathrm{W}}(X)$ if and only if $\lambda_{\mathrm{w}}(X)=\lambda_{\mathrm{s}}(X)$.

\section{The comparison with an oriented percolation}

From now on, we suppose that $X$ is countable (otherwise, $\lambda_{\mathrm{w}}^{n}=\lambda_{\mathrm{s}}^{n}=+\infty$ ). First of all, we need a coupling between $\left\{\eta_{t}\right\}_{t \geq 0}$ and $\left\{\eta_{t}^{m}\right\}_{t \geq 0}$ : think of $\left\{\eta_{t}^{m}\right\}_{t \geq 0}$ as obtained from $\left\{\eta_{t}\right\}_{t \geq 0}$ by removing all the births which cause more than $m$ particles to live on the same site. Then we need two other coupled processes. Fix $n_{0} \in \mathbb{N}$, and let $\left\{\bar{\eta}_{t}\right\}_{t \geq 0}$ be the process obtained from the BRW $\left\{\eta_{t}\right\}_{t \geq 0}$ by removing all $n$th generation particles, with $n>n_{0}$. Analogously, define $\left\{\bar{\eta}_{t}^{m}\right\}_{t \geq 0}$ from $\left\{\eta_{t}^{m}\right\}_{t \geq 0}$. Clearly, $\eta_{t} \geq \bar{\eta}_{t}, \eta_{t} \geq \eta_{t}^{m}, \eta_{t}^{m} \geq \bar{\eta}_{t}^{m}$, and $\bar{\eta}_{t} \geq \bar{\eta}_{t}^{m}$, for all $t \geq 0$. Note that, by construction, the progenies of a given particle in $\left\{\bar{\eta}_{t}\right\}_{t \geq 0}$ or $\left\{\bar{\eta}_{t}^{m}\right\}_{t \geq 0}$ live at a distance from the ancestor not larger than $n_{0}$ (and the processes go extinct almost surely).

Our proofs of the convergence of $\lambda_{\mathrm{s}}^{m}$ and $\lambda_{\mathrm{w}}^{m}$ are essentially divided into the following four steps.

Step 1. Fix a graph $(I, \mathcal{E}(I))$ such that the Bernoulli percolation on $(I, \mathcal{E}(I)) \times \overrightarrow{\mathbb{N}}$ has two phases (where we denote by $\overrightarrow{\mathbb{N}}$ the oriented graph on $\mathbb{N}$, that is, $(i, j)$ is an edge if and only if $j=i+1)$.

Note that since the (oriented) Bernoulli bond percolation on $\mathbb{Z} \times \overrightarrow{\mathbb{N}}$ and $\mathbb{N} \times \overrightarrow{\mathbb{N}}$ has two phases, it is enough to find a copy of the graph $\mathbb{Z}$ or $\mathbb{N}$ as a subgraph of $I$. This is true, for 


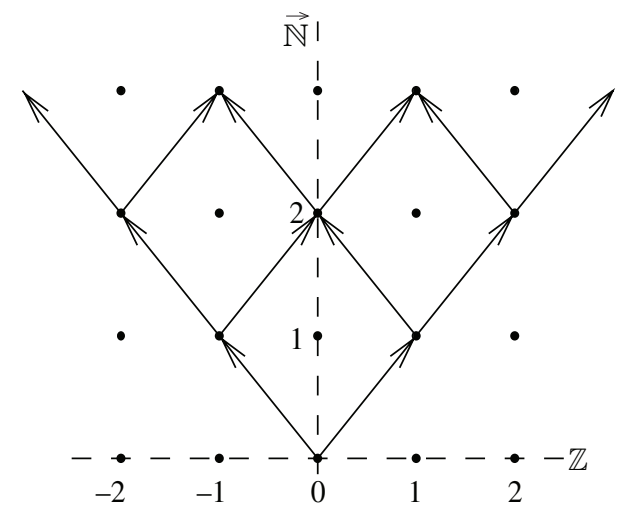

(a)

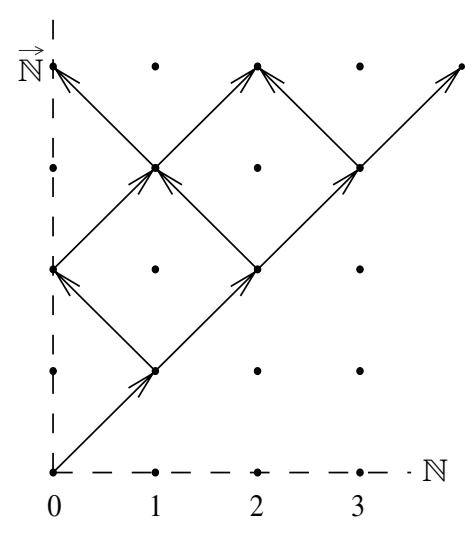

(b)

Figure 2: (a) $\mathbb{Z} \times \overrightarrow{\mathbb{N}}$. (b) $\mathbb{N} \times \overrightarrow{\mathbb{N}}$.

instance, for any infinite nonoriented graph (in this paper we choose either $I=\mathbb{Z}, I=\mathbb{N}$, or $I=X)$. Figure $2($ a) and (b) respectively show the components of the products $\mathbb{Z} \times \overrightarrow{\mathbb{N}}$ and $\mathbb{N} \times \overrightarrow{\mathbb{N}}$ containing all the vertices $y$ such that there exists a path from $(0,0)$ to $y$.

Step 2. For all $\lambda>\lambda_{\mathrm{w}}\left(\right.$ or $\left.\lambda>\lambda_{\mathrm{s}}\right)$ and every $\varepsilon>0$, there exist a collection of disjoint sets $\left\{A_{i}\right\}_{i \in I}\left(A_{i} \subset X\right.$ for all $\left.i \in I\right), \bar{t}>0$, and $k \in \mathbb{N} \backslash\{0\}$, such that, for all $i \in I$,

$$
\mathrm{P}\left(\text { for all } j:(i, j) \in \mathcal{E}(I), \sum_{x \in A_{j}} \eta_{\bar{t}}(x) \geq k \mid \eta_{0}=\eta\right)>1-\varepsilon
$$

for all $\eta$ such that $\sum_{x \in A_{i}} \eta(x)=k$ and $\eta(x)=0$ for all $x \notin A_{i}$. The same holds, for some suitable $n_{0}$, for $\left\{\bar{\eta}_{t}\right\}_{t \geq 0}$ in place of $\left\{\eta_{t}\right\}_{t \geq 0}$.

In the following sections, Step 2 will be established under certain conditions (and for suitable choices of $(I, \mathcal{E}(I)))$.

Step 3. Let $\lambda, \varepsilon,\left\{A_{i}\right\}_{i \in I}, \bar{t}$, and $k$ be chosen as in Step 2. Then, for all sufficiently large $m$, we have, for all $i \in I$,

$$
\mathrm{P}\left(\text { for all } j:(i, j) \in \mathcal{E}(I), \sum_{x \in A_{j}} \eta_{\bar{t}}^{m}(x) \geq k \mid \eta_{0}^{m}=\eta\right)>1-2 \varepsilon
$$

for all $\eta$ such that $\sum_{x \in A_{i}} \eta(x)=k$ and $\eta(x)=0$ for all $x \notin A_{i}$. The same holds, for some suitable $n_{0}$, for $\left\{\bar{\eta}_{t}^{m}\right\}_{t \geq 0}$ in place of $\left\{\eta_{t}^{m}\right\}_{t \geq 0}$.

Step 3 is a direct consequence of Step 2. Indeed, let $N_{t}$ be the total number of particles ever born in the BRW (starting from the configuration $\eta$ ) before time $t$; it is clear that $N_{t}$ is a process bounded above by a branching process with birth rate $K \lambda$, death rate 0 , and starting with $k$ particles. If $N_{0}<+\infty$ almost surely then, for all $t>0$, we have $N_{t}<+\infty$ almost surely; hence, for all $t>0$ and $\varepsilon>0$, there exists $n(t, \varepsilon)$ such that, for all $i \in I$,

$$
\mathrm{P}\left(N_{t} \leq n(t, \varepsilon) \mid \eta_{0}=\eta\right)>1-\varepsilon
$$

for all $\eta$ such that $\sum_{x \in A_{i}} \eta(x)=k$ and $\eta(x)=0$ for all $x \notin A_{i}$. Define $\bar{n}=n(\bar{t}, \varepsilon)$. We note 
that, for any event $A$ such that $\mathrm{P}\left(A \mid \eta_{0}=\eta\right)>1-\varepsilon$, we have

$$
\mathrm{P}\left(A \mid N_{t} \leq \bar{n}, \eta_{0}=\eta\right) \geq \mathrm{P}\left(A, N_{t} \leq \bar{n} \mid \eta_{0}=\eta\right) \geq 1-2 \varepsilon .
$$

Choose $m \geq \bar{n}$. Then $\eta_{t}=\eta_{t}^{m}$ for all $t \leq \bar{t}$ on $\left\{N_{\bar{t}} \leq \bar{n}\right\}=\bigcap_{t \leq \bar{t}}\left\{N_{t} \leq \bar{n}\right\}$. Thus, (4.1) and (4.3) imply (4.2). The claim for $\bar{\eta}_{t}^{m}$ is proven analogously.

Step 4. For all $\lambda>\lambda_{\mathrm{w}}\left(\right.$ or $\left.\lambda>\lambda_{\mathrm{s}}\right)$ and every $\varepsilon>0$, for all sufficiently large $m$, there exists a one-dependent oriented percolation on $I \times \overrightarrow{\mathbb{N}}$ (with probability $1-2 \varepsilon$ of simultaneously opening all edges from a vertex and with probability $2 \varepsilon$ of opening no edges) such that the probability of survival of the $B R W_{m}$ (starting at time 0 from a configuration $\eta$ such that $\sum_{x \in A_{i_{0}}} \eta(x)=k$ and $\eta(x)=0$ for all $\left.x \notin A_{i_{0}}\right)$ is larger than the probability that there exists an infinite cluster containing $\left(i_{0}, 0\right)$.

In order to prove Step 4 using Step 3, we need another auxiliary process, namely $\left\{\widehat{\eta}_{t}\right\}_{t \geq 0}$ defined from $\bar{\eta}_{t}$ by suppressing all newborns after the $\bar{n}$ th particle is born. If $m \geq \bar{n}$ then $\widehat{\eta}_{t} \leq \bar{\eta}_{t}^{m} \leq \eta_{t}^{m}$ for all $t \geq 0$, and, for all $t \leq \bar{t}, \widehat{\eta}_{t}=\bar{\eta}_{t}^{m}$ on $\left\{N_{\bar{t}} \leq \bar{n}\right\}$.

Consider an edge $((i, n),(j, n+1))$ in $(I, \mathcal{E}(I)) \times \overrightarrow{\mathbb{N}}$. Let it be open if $\eta_{t}^{m}$ has at least $k$ individuals in $A_{i}$ at time $n \bar{t}$ and in $A_{j}$ at time $(n+1) \bar{t}$. Thus, the probability of weak survival of $\eta_{t}^{m}$ is bounded from below by the probability that there exists an infinite cluster containing $\left(i_{0}, 0\right)$ in this percolation on $I \times \overrightarrow{\mathbb{N}}$, and, if $A_{i_{0}}$ is finite, the probability of strong survival is bounded from below by the probability that the cluster contains infinitely many points in $\left\{\left(i_{0}, l\right): l \in \mathbb{N}\right\}$ (we suppose that we start with $k$ particles in $A_{i_{0}}$ ). Let $v_{1}$ be the associated percolation measure. Unfortunately, this percolation is neither independent nor one-dependent. In fact, the opening procedure of the edges $((i, n),(j, n+1))$ and $\left(\left(i_{1}, n\right),\left(j_{1}, n+1\right)\right)$ may depend on two different progenies of particles overlapping on a vertex $x_{0}$. This may cause dependence since if in $x_{0}$ there are already $m$ particles then newborns are not allowed.

To avoid this difficulty, we will choose $m$ sufficiently large and consider another percolation on $I \times \overrightarrow{\mathbb{N}}$. Let $\widehat{\eta}_{i_{0}, 0, t}$ (constructed from $\eta_{t}$ with the usual removal rules) start with $k$ particles in $A_{i_{0}}$ : we open all edges $\left(i_{0}, 0\right) \rightarrow(j, 1)$ if $\widehat{\eta}_{i_{0}, 0, \bar{t}}$ has at least $k$ particles in $A_{j}$. With a slight abuse of notation, we define $\widehat{\eta}_{t}:=\widehat{\eta}_{i_{0}, 0, t}$ for all $t \in(0, \bar{t}]$. We construct the process $\left\{\widehat{\eta}_{t}\right\}_{t \geq 0}$ by iteration on the time intervals $(n \bar{t},(n+1) \bar{t}]$, where $n \in \mathbb{N}$. We constructed it above for the case in which $n=0$.

Suppose that we constructed $\left\{\widehat{\eta}_{t}\right\}_{t \geq 0}$ for $t \in[0, n \bar{t}]$; we construct it now for $t \in(n \bar{t},(n+1) \bar{t}]$. For all $j$ such that $\widehat{\eta}_{n \bar{t}}$ has at least $k$ particles in $A_{j}$, we start a process $\left\{\widehat{\eta}_{j, n, t}\right\}_{t \geq 0}$ with initial configuration given by $k$ particles in $A_{j}$ (chosen among the particles that $\widehat{\eta}_{n \bar{t}}$ has in $A_{j}$ ) and 0 elsewhere. Note that the $\left\{\widehat{\eta}_{j, n, t}\right\}_{j}$ are conditionally independent with respect to $\widehat{\eta}_{n \bar{t}}$. As before, we define $\widehat{\eta}_{t}:=\sum_{j} \widehat{\eta}_{j, n, t-n \bar{t}}$ for all $t \in(n \bar{t},(n+1) \bar{t}]$.

Choosing $m$ sufficiently large we have $\widehat{\eta}_{t} \leq \bar{\eta}_{t}^{m} \leq \eta_{t}^{m}$. Indeed, it is enough to choose $m \geq 2 \bar{n} H$, where $H \in \mathbb{N}$ is the supremum over $x$ of the number of paths of length $n_{0}$ which contain a fixed vertex $x$ and $\bar{n}$ is the same as in (4.3); $H$ is finite since $(X, \mu)$ is with bounded geometry. To the process $\left\{\widehat{\eta}_{t}\right\}_{t \geq 0}$ we associate a percolation $v_{2}$, such that $v_{1} \geq v_{2}$, in the following way: we open the edge from $(i, n)$ to $(j, n+1)$ if $\widehat{\eta}_{i, n, \bar{t}}$ has at least $k$ particles in $A_{j}$.

By (4.2), the probability of opening all edges from $(i, n)$ according to $v_{2}$ (conditioned to the event ' $\left(i_{0}, 0\right)$ is connected to $(i, n)$ ') is at least $1-2 \varepsilon$. We observe that the set of open edges from $(i, n)$ depends only on the progenies of the $k$ particles alive in $A_{i}$ at time $n \bar{t}$ (hence on $\widehat{\eta}_{i, t}$, which are conditionally independent with respect to $\left.\widehat{\eta}_{n \bar{t}}\right)$.

We construct a one-dependent percolation (i.e. a percolation where the openings of edges from different vertices are independent) $\nu_{3}$ as follows: we simultaneously open all the edges 
from $(i, n)$ with probability $1-2 \varepsilon$ and no edge with probability $2 \varepsilon$. Finally, the percolation processes $\nu_{2}$ and $\nu_{3}$ can be coupled in such a way that, almost surely, the cluster $\Omega_{3}$ containing the starting point $\left(i_{0}, 0\right)$ in percolation $\nu_{3}$ is a subset of the cluster $\Omega_{2}$ containing $\left(i_{0}, 0\right)$ in percolation $\nu_{2}$. Indeed, if $\beta_{n}:=\{(j, m) \in I \times \overrightarrow{\mathbb{N}}: m \leq n\}$ then it is not difficult to prove by induction on $n$ that $\mathrm{P}\left(\Omega_{3} \cap \beta_{n} \subseteq \Omega_{2} \cap \beta_{n}\right)=1$ (where $\mathrm{P}$ is the probability associated to the coupled processes). Hence, we find that the probability of existence of an infinite cluster containing $\left(i_{0}, 0\right)$ in percolation $v_{2}$ is larger than or equal to the probability of the same event in percolation $v_{3}$. This concludes Step 4 .

We note that the trick is to fix a suitable $(I, \mathcal{E}(I))$ and prove Step 2 for all $\lambda>\lambda_{\mathrm{w}}$. Then, by Steps 1 and 4, for all sufficiently large $m$, the $\lambda-\mathrm{BRW}_{m}$ survives with positive probability and we deduce that $\lambda_{\mathrm{w}}^{m} \rightarrow \lambda_{\mathrm{w}}$ as $m \rightarrow \infty$. On the other hand, to show that $\lambda_{\mathrm{s}}^{m} \rightarrow \lambda_{\mathrm{s}}$ as $m \rightarrow \infty$, we need to prove Step 2 with a choice of at least one $A_{i}$ finite, say $A_{i_{0}}$, and $I$ containing a copy of $\mathbb{Z}$ or $\mathbb{N}$ as a subgraph. Indeed, the infinite open cluster in a supercritical Bernoulli bond percolation in $\mathbb{Z} \times \overrightarrow{\mathbb{N}}$ or $\mathbb{N} \times \overrightarrow{\mathbb{N}}$ with probability 1 has an infinite intersection with the set $\{(0, n): n \in \mathbb{N}\}$. As a consequence, in the supercritical case we have, with positive probability, an infinite open cluster in $\mathbb{Z} \times \overrightarrow{\mathbb{N}}$ (or $\mathbb{N} \times \overrightarrow{\mathbb{N}}$ ) which contains the origin $(0,0)$ and infinite vertices of the set $\{(0, n): n \geq 0\}$. This implies (again by Steps 3 and 4 ) that, with positive probability, the $\lambda$-BRW $m$ starting with $k$ particles in $A_{i_{0}}$ has particles alive in $A_{i_{0}}$ at arbitrarily large times. Being the cardinality of $A_{i_{0}}$ finite yields the conclusion.

Remark 4.1. The previous set of steps represents the skeleton of the proofs of Theorems 5.1 and 6.2, below. In Theorem 6.1, below, we need a generalization of this approach. We sketch here the main differences. We choose an oriented graph $(W, \mathcal{E}(W))$ and a family of subsets of $X,\left\{A_{(i, n)}\right\}_{(i, n) \in W}$, such that

- $W$ is a subset of the set $\mathbb{Z} \times \mathbb{N}$ (note that this is an inclusion between sets, not between graphs);

- for all $n \in \mathbb{N},\left\{A_{(i, n)}\right\}_{i:(i, n) \in W}$ is a collection of disjoint subsets of $X$;

- $(i, n) \rightarrow(j, m)$ implies that $m=n+1$.

The analog of Step 2 is the following: for all sufficiently large $\lambda$ (for instance, $\lambda>\lambda_{\mathrm{s}}$ or $\lambda>\lambda_{\mathrm{w}}$ ) and every $\varepsilon>0$, there exist $\bar{t}>0$ and $k \in \mathbb{N}$ such that, for all $n \in \mathbb{N}, i \in \mathbb{Z}$, and all $\eta$ such that $\sum_{x \in A_{(i, n)}} \eta(x)=k$,

$$
\mathrm{P}\left(\text { for all } j:(i, n) \rightarrow(j, n+1), \sum_{x \in A_{(j, n)}} \eta_{(n+1) \bar{t}}(x) \geq k \mid \eta_{n \bar{t}}=\eta\right)>1-\varepsilon
$$

Step 3 is the same as before and the percolation described in Step 4 now concerns the graph $(W, \mathcal{E}(W))$ (instead of $(I, \mathcal{E}(I)) \times \overrightarrow{\mathbb{N}})$.

\section{Approximation of $\lambda_{\mathrm{s}}$ by $\lambda_{\mathrm{s}}^{m}$}

We choose the initial configuration as $\delta_{o}$ (where $o$ is a fixed vertex in $X$ ), and we first study the expected value of the number of individuals in one site at some time, that is, $\mathrm{E}^{\delta_{o}}\left(\eta_{t}(x)\right)$. This is done using the semigroup $S_{t}$; indeed, if we define the evaluation maps $e_{x}(\eta):=\eta(x)$ for any $\eta \in \mathbb{N}^{X}$ and $x \in X$, then $\mathrm{E}^{\eta}\left(\eta_{t}(x)\right)=S_{t} e_{x}(\eta)$. 
By standard theorems (see [8, Chapter 1] for the compact case, or, since $\mathbb{N}^{X}$ is not locally compact, [3] and [14]),

$$
\left.\frac{\mathrm{d}}{\mathrm{d} t} S_{t} e_{x}\right|_{t=t_{0}}=S_{t_{0}} \mathcal{L} e_{x}
$$

from which we deduce that

$$
\frac{\mathrm{d}}{\mathrm{d} t} \mathrm{E}^{\eta}\left(\eta_{t}(x)\right)=-\mathrm{E}^{\eta}\left(\eta_{t}(x)\right)+\lambda \sum_{z \in X} \mu(z, x) \mathrm{E}^{\eta}\left(\eta_{t}(z)\right) .
$$

It is not difficult to verify that

$$
\mathrm{E}^{\delta_{x_{0}}}\left(\eta_{t}(x)\right)=\sum_{n=0}^{\infty} \mu^{(n)}\left(x_{0}, x\right) \frac{(\lambda t)^{n}}{n !} \mathrm{e}^{-t} .
$$

Remark 5.1. For all $x, x_{0} \in X$, all $\lambda>0$, and $n \in \mathbb{N}$,

$$
\mathrm{E}^{\delta_{x_{0}}}\left(\eta_{n}(x)\right) \geq \mu^{(n)}\left(x_{0}, x\right) \frac{\lambda^{n} n^{n}}{n !} \mathrm{e}^{-n} \sim \mu^{(n)}\left(x_{0}, x\right) \frac{\lambda^{n}}{\sqrt{2 \pi n}},
$$

and the same inequality holds if $n_{0} \geq n$, with $\bar{\eta}_{n}$ in place of $\eta_{n}$.

Depending on $\lambda$, we may characterize the behavior of the expected number of descendants at a fixed site.

Lemma 5.1. Let us fix $x \in X$. If $\lambda<R_{\mu}$ then $\lim _{t \rightarrow+\infty} \mathrm{E}^{\delta_{x_{0}}}\left(\eta_{t}(x)\right)=0$; if $\lambda>R_{\mu}$ then $\lim _{t \rightarrow+\infty} \mathrm{E}^{\delta_{x_{0}}}\left(\eta_{t}(x)\right)=+\infty$.

Proof. Let $\lambda<R_{\mu}$. For all $\varepsilon>0$, there exists $n_{0}$ such that $\mu^{(n)}\left(x_{0}, x\right)<1 /\left(R_{\mu}-\varepsilon\right)^{n}$ for all $n \geq n_{0}$. If $\varepsilon=\left(R_{\mu}-\lambda\right) / 2$ then $\lambda^{n} \mu^{(n)}\left(x_{0}, x\right) \leq\left(2 \lambda /\left(R_{\mu}+\lambda\right)\right)^{n}$ for all $n \geq n_{0}$; hence, $\mathrm{E}^{\delta_{x_{0}}}\left(\eta_{t}(x)\right) \leq Q(t) \mathrm{e}^{-t}+\exp \left[-t\left(R_{\mu}-\lambda\right) /\left(R_{\mu}+\lambda\right)\right] \rightarrow 0$ as $t \rightarrow \infty(Q$ is a polynomial of degree at most $\left.n_{0}-1\right)$.

Let $\lambda>R_{\mu}$. If $k, r \in \mathbb{N}$ are such that $\mu^{(k)}\left(x_{0}, x\right)>0$ and $\mu^{(i r)}(x, x)>0$ for all $i \in \mathbb{N}$, then

$$
\mathrm{E}^{\delta_{x_{0}}}\left(\eta_{t}(x)\right) \geq \mu^{(k)}\left(x_{0}, x\right) \mathrm{e}^{-t} \sum_{i=0}^{\infty} \frac{\mu^{(i r)}(x, x)(\lambda t)^{i r+k}}{(r i+k) !} .
$$

Let us define $a_{n}:=\mu^{(n r)}(x, x)$; clearly, $a_{n+m} \geq a_{n} a_{m}$ and $R_{\mu}=1 / \lim _{n \rightarrow \infty} \sqrt[n r]{a_{n}}$ (since $\left\{a_{n}\right\}_{n \in \mathbb{N}}$ is supermultiplicative, the limit exists). then

We now prove that, for any nonnegative, supermultiplicative sequence $\left\{a_{n}\right\}_{n \in \mathbb{N}}$, if $\lambda>R_{\mu}$

$$
\lim _{t \rightarrow \infty} \mathrm{e}^{-t} \sum_{i=0}^{\infty} a_{i} \frac{(\lambda t)^{i r+k}}{(i r+k) !}=+\infty .
$$

Indeed, let $n_{0}$ be such that $a_{n_{0}} \geq 2^{r n_{0}}(R+\lambda)^{-r n_{0}}$, and define

$$
f(t):=\mathrm{e}^{-t} \sum_{i=0}^{\infty} \frac{a_{i}(\lambda t)^{i r+k}}{(i r+k) !}
$$


Clearly,

$$
f(t) \geq \mathrm{e}^{-t}\left(\frac{R+\lambda}{2}\right)^{k} \sum_{i=0}^{\infty} \frac{\left(\lambda^{\prime} t\right)^{i n_{0} r+k}}{\left(i n_{0} r+k\right) !}
$$

where $\lambda^{\prime}=2 \lambda /(R+\lambda)>1$. For all $i \in \mathbb{N}$, we have

$$
\frac{\left(\lambda^{\prime} t\right)^{i n_{0} r+k}}{\left(i n_{0} r+k\right) !}+\frac{\left(\lambda^{\prime} t\right)^{i n_{0} r+k+1}}{\left(i n_{0} r+k+1\right) !}+\cdots+\frac{\left(\lambda^{\prime} t\right)^{i n_{0} r+k+n_{0} r-1}}{\left(i n_{0} r+k+n_{0} r-1\right) !} \leq \frac{\left(\lambda^{\prime} t\right)^{i n_{0} r+k}}{\left(i n_{0} r+k\right) !} \frac{\left(\lambda^{\prime} t\right)^{n_{0} r}-1}{\lambda^{\prime} t-1}
$$

thus,

$$
f(t) \geq \mathrm{e}^{-t}\left(\frac{R+\lambda}{2}\right)^{k} \frac{\lambda^{\prime} t-1}{\left(\lambda^{\prime} t\right)^{n_{0} r}-1} \sum_{i=k}^{\infty} \frac{\left(\lambda^{\prime} t\right)^{i}}{i !} \rightarrow \infty
$$

exponentially if $t \rightarrow \infty$ (since $\left.\lambda^{\prime}>1\right)$.

In the following lemma we prove that, when $\lambda>R_{\mu}$, if at time 0 we have one individual at each of the $l$ sites $x_{1}, \ldots, x_{l}$, then, given any choice of $l$ sites $y_{1}, \ldots, y_{l}$, after some time the expected number of descendants in $y_{i}$ of the individual in $x_{i}$ exceeds 1 for all $i=1, \ldots, l$. The proof is easy and thus we omit it.

Lemma 5.2. Let us consider a finite set of couples $\left\{\left(x_{j}, y_{j}\right)\right\}_{j=0}^{l}$; if $\lambda>R_{\mu}$ then there exists $t=t(\lambda)>0$ such that $\mathrm{E}^{\delta_{x_{j}}}\left(\eta_{t}\left(y_{j}\right)\right)>1$ for all $j=0,1, \ldots, l$. Moreover, $\mathrm{E}^{\delta_{x_{j}}}\left(\bar{\eta}_{t}\left(y_{j}\right)\right)>1$ when $n_{0}$ is sufficiently large.

So far, we have obtained results on the expected number of individuals; now we show that, when $\lambda>R_{\mu}$, for all sufficiently large $k \in \mathbb{N}$, given $k$ particles in a site $x$ at time 0 , 'typically' (i.e. with arbitrarily large probability) after some time we will have at least $k$ individuals in each site of a fixed finite set $Y$. Analogously, starting with $l$ colonies of size $k$ (in sites $x_{1}, \ldots, x_{l}$, respectively), each of them will, after a sufficiently long time, spread at least $k$ descendants in every site of a corresponding (finite) set of sites $Y_{i}$.

Lemma 5.3. Suppose that $\lambda>R_{\mu}$.

(i) Let us fix $x \in X$, let $Y$ be a finite subset of $X$, and let $\varepsilon>0$. Then there exist $t=$ $t(\lambda, x)>0$ (independent of $\varepsilon$ ) and $k(\varepsilon, x, Y, \lambda)$ such that, for all $k \geq k(\varepsilon, x, Y, \lambda)$,

$$
\mathrm{P}\left(\bigcap_{y \in Y}\left(\eta_{t}(y) \geq k\right) \mid \eta_{0}(x)=k\right)>1-\varepsilon .
$$

The claim also holds with $\left\{\bar{\eta}_{t}\right\}_{t \geq 0}$ in place of $\left\{\eta_{t}\right\}_{t \geq 0}$ when $n_{0}$ is sufficiently large.

(ii) Let us fix a finite set of vertices $\left\{x_{i}\right\}_{i=1, \ldots, m}$ and a collection of finite sets $\left\{Y_{i}\right\}_{i=1, \ldots, l}$ of vertices of $X$, and let $\varepsilon>0$. Then there exist $t=t\left(\lambda,\left\{x_{i}\right\},\left\{Y_{i}\right\}\right)$ (independent of $\varepsilon$ ) and $k\left(\varepsilon,\left\{x_{i}\right\},\left\{Y_{i}\right\}, \lambda\right)$ such that, for all $i=1, \ldots, l$ and $k \geq k\left(\varepsilon,\left\{x_{i}\right\},\left\{Y_{i}\right\}, \lambda\right)$,

$$
\mathrm{P}\left(\bigcap_{y \in Y_{i}}\left(\eta_{t}(y) \geq k\right) \mid \eta_{0}\left(x_{i}\right)=k\right)>1-\varepsilon .
$$

The claim also holds with $\left\{\bar{\eta}_{t}\right\}_{t \geq 0}$ in place of $\left\{\eta_{t}\right\}_{t \geq 0}$ when $n_{0}$ is sufficiently large. 
Proof. (i) If we denote by $\left\{\xi_{t}\right\}_{t}$ the branching process starting from $\xi_{0}=\delta_{x}$ then, by Lemma 5.2, we can choose $t$ such that $\mathrm{E}^{\delta_{x}}\left(\xi_{t}(y)\right)>1$ for all $y \in Y$. We can write $\eta_{t}(y)=$ $\sum_{j=1}^{k} \xi_{t, j}(y)$, where $\xi_{t, j}(y)$ denotes the number of descendants in $y$ of the $j$ th initial particle; note that $\left\{\xi_{t, j}(y)\right\}_{j \in \mathbb{N}}$ is an independent and identically distributed family with $\mathrm{E}\left(\xi_{t, j}(y)\right)=$ $\mathrm{E}^{\delta_{x}}\left(\xi_{t}(y)\right)$ and $\operatorname{var}\left(\xi_{t, j}(y)\right)=: \sigma_{t, y}^{2}$. Since $\xi_{t, j}$ is stochastically dominated by a continuoustime branching process with birth rate $\lambda$, it is clear that $\sigma_{t, y}^{2}<+\infty$. Thus, by the central limit theorem, given any $\delta>0$, if $k$ is sufficiently large,

$$
\delta \geq\left|\mathrm{P}\left(\sum_{j=1}^{k} \xi_{t, j}(y) \geq z\right)-1+\Phi\left(\frac{z-k \mathrm{E}^{\delta_{x}}\left(\xi_{t}(y)\right)}{\sqrt{k} \sigma_{t, y}}\right)\right|
$$

uniformly with respect to $z \in \mathbb{R}$, where $\Phi$ is the cumulative distribution function of the standard normal. Whence there exists $k(\delta, x, y)$ such that, for all $k \geq k(\delta, x, y)$,

$$
\mathrm{P}\left(\eta_{t}(y) \geq k\right) \geq 1-\Phi\left(\sqrt{k} \frac{1-\mathrm{E}^{\delta_{x}}\left(\eta_{t}(y)\right)}{\sigma_{t, y}}\right)-\delta \geq 1-2 \delta,
$$

since $\sqrt{k}\left(1-\mathrm{E}^{\delta_{x}}\left(\eta_{t}(y)\right)\right) / \sigma_{t, y} \rightarrow-\infty$ as $k \rightarrow+\infty$. Take $k(\delta, x, Y):=\max _{y \in Y} k(\delta, x, y)<$ $+\infty$, and let $D$ be the cardinality of $Y$. Hence, for all $k \geq k_{x}$,

$$
\mathrm{P}\left(\bigcap_{y \in Y}\left(\eta_{t}(y) \geq k\right) \mid \eta_{0}(x)=k\right) \geq 1-2 D \delta .
$$

The assertion for $\bar{\eta}_{t}$ follows from Lemma 5.2.

(ii) Let $\left\{\xi_{t}\right\}_{t \geq 0}$ be as before, and choose $t$ such that $\mathrm{E}^{\delta_{x_{i}}}\left(\xi_{t}(y)\right)>1$ for all $y \in Y_{i}$ and all $i=1, \ldots, l$. According to part (i), we fix $k_{i}$ such that, for all $k \geq k_{i}$,

$$
\mathrm{P}\left(\bigcap_{y \in Y_{i}}\left(\eta_{t}(y) \geq k\right) \mid \eta_{0}\left(x_{i}\right)=k\right) \geq 1-\varepsilon .
$$

Take $k \geq \max _{i=1, \ldots, l} k_{i}$ to conclude. The assertion for $\bar{\eta}_{t}$ follows from Lemma 5.2.

Remark 5.2. Note that Remark 5.1 and Lemmas 5.2 and 5.3 can be restated for the process $\left\{\eta_{t}^{\bar{m}}\right\}_{t \geq 0}$ if $m$ is sufficiently large.

We say that $(X, \mu)$ is quasi-transitive if there exists a finite partition of $X$ such that, for all couples $(x, y)$ in the same class, there exists a bijection $\gamma$ on $X$ satisfying $\gamma(x)=y$ and, for all $a, b \in X, \mu(\gamma(a), \gamma(b))=\mu(a, b)$ (when the last condition holds, we say that $\mu$ is $\gamma$-invariant). In particular, if $\mu(x, y)=p(x, y)$, where $P$ is the simple random walk on $X$, then it is $\gamma$-invariant for any automorphism $\gamma$. Roughly speaking, if $\mu$ is $\gamma$-invariant then the graph is identical seen from $x$ and $\gamma(x)$.

Theorem 5.1. Suppose that at least one of the following conditions holds:

(i) $(X, \mu)$ is quasi-transitive;

(ii) $(X, \mu)$ is connected and there exists a bijection $\gamma$ on $X$ such that

(a) $\mu$ is $\gamma$-invariant;

(b) for some $x_{0} \in X$, we have $x_{0}=\gamma^{n} x_{0}$ if and only if $n=0$. 
Then

$$
\lim _{m \rightarrow+\infty} \lambda_{\mathrm{s}}^{m}=\lambda_{\mathrm{s}} \geq \lim _{m \rightarrow+\infty} \lambda_{\mathrm{w}}^{m} \geq \lambda_{\mathrm{w}} .
$$

Moreover, if $\lambda_{\mathrm{s}}=\lambda_{\mathrm{w}}$ then $\lambda_{\mathrm{w}}^{m} \downarrow \lambda_{\mathrm{w}}$ as $m \rightarrow+\infty$.

Proof. Remember that $\lambda_{\mathrm{s}}=R_{\mu}$.

(i) Let us collect one vertex from each orbit into the (finite) set $\left\{x_{i}\right\}_{i=1, \ldots, l}$, and let $Y_{i}:=\{y \in$ $\left.X: x_{i} \rightarrow y\right\}$. Fix $\lambda>R_{\mu}, I=X, \mathcal{E}(I)=\{(x, y):(x, y) \in \mathcal{E}(X)$ or $(y, x) \in \mathcal{E}(X)\}$, and $A_{x}=\{x\}$. Note that $(I, \mathcal{E}(I))$ coincides with $(X, \mathcal{E}(X))$ if the latter is nonoriented. By these choices, Lemma 5.3 yields Step 2. To prove that the percolation on $(I, \mathcal{E}(I)) \times \overrightarrow{\mathbb{N}}$ has two phases (that is, $(I, \mathcal{E}(I))$ is a suitable choice for Step 1), we note that the existence of the supercritical phase for the Bernoulli percolation on $X \times \overrightarrow{\mathbb{N}}$ follows from the fact that the graph $\mathbb{N}$ is a subgraph of $X$. Moreover, in the supercritical Bernoulli percolation on $\mathbb{N} \times \overrightarrow{\mathbb{N}}$, with positive probability, the infinite open cluster contains $(0,0)$ and intersects the $y$-axis infinitely often. Hence, by Steps 3 and 4, for all sufficiently large $m, \lambda_{\mathrm{s}}^{m} \leq \lambda$, and this yields the result.

(ii) Fix $\lambda>R_{\mu}$; by Lemma 5.3 , for sufficiently large $n_{0}$,

$$
\mathrm{P}\left(\bar{\eta}_{t}(\gamma x) \geq k \mid \bar{\eta}_{0}(x)=k\right)>1-\varepsilon \quad \text { and } \quad \mathrm{P}\left(\bar{\eta}_{t}(x) \geq k \mid \bar{\eta}_{0}(\gamma x)=k\right)>1-\varepsilon .
$$

This implies that

$\mathrm{P}\left(\bar{\eta}_{t}\left(\gamma^{n} x\right) \geq k \mid \bar{\eta}_{0}\left(\gamma^{n-1} x\right)=k\right)>1-\varepsilon \quad$ and $\quad \mathrm{P}\left(\bar{\eta}_{t}\left(\gamma^{n-1} x\right) \geq k \mid \bar{\eta}_{0}\left(\gamma^{n} x\right)=k\right)>1-\varepsilon$

for all $n \in \mathbb{Z}$ since $\mu$ is $\gamma$-invariant; again, Steps 3 and 4 yield $\lambda_{\mathrm{s}}^{m} \leq \lambda$ (for sufficiently large $m$ ) and the claim (here $I=\mathbb{Z}$ and $A_{i}=\left\{\gamma^{i} x_{0}\right\}$ ).

\section{Approximation of $\lambda_{w}$ by $\lambda_{w}^{m}$}

From now on we set $\mu(x, y)=p(x, y)$, where $P$ is a stochastic matrix. We stress that in this case $\lambda_{\mathrm{w}}=1$. We are concerned with the question of whether $\lambda_{\mathrm{w}}^{m} \downarrow \lambda_{\mathrm{w}}=1$ or not. Under the hypotheses of Theorem 5.1, this is the case when the BRW has no pure weak phase (i.e. $R=1$ ). The interesting case is $R>1$. The most natural examples are drifting random walks on $\mathbb{Z}^{d}$ and the simple random walk on homogeneous trees. In both cases we show that $\lambda_{\mathrm{w}}^{m} \rightarrow \lambda_{\mathrm{w}}$ as $m \rightarrow \infty$.

Theorem 6.1. Let $P$ be a random walk on $\mathbb{Z}$ such that $p(i, i+1)=p, p(i, i-1)=q$, and $p(i, i)=1-p-q$, for all $i \in \mathbb{Z}$. Then $\lim _{m \rightarrow+\infty} \lambda_{\mathrm{w}}^{m}=1=\lambda_{\mathrm{w}}$.

Proof. We consider $\alpha, \beta \in(0,1), \alpha \leq \beta \leq(1+\alpha) / 2$, and write

$$
\begin{aligned}
p^{(n)}(0, \alpha n) & \\
\quad & =\sum_{i=\alpha n}^{(1+\alpha) n / 2}\left(\begin{array}{c}
n \\
i, i-\alpha n, n-2 i+\alpha n
\end{array}\right) p^{i} q^{i-\alpha n}(1-p-q)^{n-2 i+\alpha n} \\
& \geq\left(\begin{array}{c}
n n,(\beta-\alpha) n,(1-2 \beta+\alpha) n
\end{array}\right) p^{\beta n} q^{(\beta-\alpha) n}(1-p-q)^{(1-2 \beta+\alpha) n} \\
& \sim \frac{1}{2 \pi n \sqrt{\beta(\beta-\alpha)(1-2 \beta+\alpha)}}\left(\frac{p^{\beta} q^{\beta-\alpha}(1-p-q)^{1-2 \beta+\alpha}}{\beta^{\beta}(\beta-\alpha)^{\beta-\alpha}(1-2 \beta+\alpha)^{1-2 \beta+\alpha}}\right)^{n} \text { as } n \rightarrow \infty
\end{aligned}
$$


Thus, if $\lambda>1, \mathrm{E}^{\delta_{0}}\left(\eta_{n}(\alpha n)\right)$ is bounded from below by a quantity which is asymptotic to

$$
\frac{1}{(2 \pi n)^{3 / 2} \sqrt{\beta(\beta-\alpha)(1-2 \beta+\alpha)}}\left(g_{\lambda}(\alpha, \beta)\right)^{n},
$$

where

$$
g_{\lambda}(\alpha, \beta)=\frac{\lambda p^{\beta} q^{\beta-\alpha}(1-p-q)^{1-2 \beta+\alpha}}{\beta^{\beta}(\beta-\alpha)^{\beta-\alpha}(1-2 \beta+\alpha)^{1-2 \beta+\alpha}} .
$$

Note that $g_{\lambda}(p-q, p)=\lambda$; thus, we can find $\alpha_{1}<\alpha_{2} \leq \beta_{1}<\beta_{2}$ (with $\beta_{i} \leq\left(1+\alpha_{i}\right) / 2$, $i=1,2)$ such that $g_{\lambda}(x, y)>1$ for all $(x, y) \in\left[\alpha_{1}, \alpha_{2}\right] \times\left[\beta_{1}, \beta_{2}\right]$. By taking $n=\bar{n}$ sufficiently large we can find three distinct integers, $d_{1}, d_{2}$, and $d_{3}$, such that $\alpha_{1} n \leq d_{1}<d_{2} \leq \alpha_{2} n$, $\beta_{1} n \leq d_{3} \leq \beta_{2} n$, and $g_{\lambda}\left(d_{l} / n, d_{3} / n\right)>1, l=1,2$.

By the same reasoning as in Lemma 5.3 we find that, for all $\lambda>1$ and $\varepsilon>0$, there exist $\bar{t}$ and $k=k(\varepsilon, \lambda)$ such that, for all $i \in \mathbb{Z}$ and all sufficiently large $n_{0}$,

$$
\mathrm{P}\left(\bar{\eta}_{\bar{t}}(i+j) \geq k, j=d_{1}, d_{2} \mid \bar{\eta}_{0}=k \delta_{i}\right)>1-\varepsilon .
$$

Since $k$ and $\bar{t}$ are independent of $i$, we have proved the general version of Step 2 as stated in Remark 4.1 (where $W=\left\{a\left(d_{1}, 1\right)+b\left(d_{2}, 1\right): a, b \in \mathbb{N}\right\}, A_{(i, n)}=\{i\}$, and $(i, n) \rightarrow(j, n+1)$ if and only if $j-i=d_{1}$ or $j-i=d_{2}$ ).

In view of Corollary 6.1 and Theorem 6.2, below, it is useful to introduce the concept of local isomorphism, which allows us to extend some results from $\mathbb{Z}$ to more general graphs. Given two weighted graphs $(X, \mu)$ and $(I, v)$, we say that a map $f: X \rightarrow I$ is a local isomorphism of $X$ on $I$ if, for all $x \in X$ and $i \in I$, we have $\sum_{z \in f^{-1}(i)} \mu(x, z)=v(f(x), i)$.

In this case it is clear that, if we consider the partition of $X$ given by $\left\{A_{i}\right\}_{i \in I}$, where $A_{i}:=$ $f^{-1}(i)$, we can easily compute the expected number of particles alive at time $t$ in $A_{i}$ starting from a single particle alive in $x$ at time 0 :

$$
\sum_{z \in A_{i}} \mathrm{E}_{\mu}^{\delta_{x}}\left(\eta_{t}(z)\right)=\mathrm{E}_{v}^{\delta_{f(x)}}\left(\xi_{t}(i)\right)
$$

(where $\left\{\xi_{t}\right\}_{t \geq 0}$ is a branching random walk on $(I, v)$ ), since $\sum_{z \in f^{-1}(i)} \mu^{(n)}(x, z)=v^{(n)}(f(x), i)$ for all $n \in \mathbb{N}$. We note that the latter depends only on $f(x)$ and $i$. As a consequence, $R_{\mu} \geq R_{\nu}$.

Corollary 6.1. If $P$ is a translation invariant random walk on $\mathbb{Z}^{d}$ then $\lim _{k \rightarrow+\infty} \lambda_{\mathrm{w}}^{k}=1=\lambda_{\mathrm{w}}$.

Proof. Let $\left\{Z_{n}\right\}_{n \in \mathbb{N}}$ be a realization of the random walk, and let $A_{i}=\left\{x \in \mathbb{Z}^{d}: x(1)=i\right\}$. Note that

$$
\mathrm{P}\left(Z_{n+1} \in A_{j} \mid Z_{n}=w\right)=\tilde{p}(i, j) \text { for all } w \in A_{i},
$$

where $\widetilde{P}$ is a random walk on $\mathbb{Z}$ with $p=p\left(0, e_{1}\right)$ and $q=p\left(0,-e_{1}\right)$. Using (6.1) and reasoning as in the proof of the previous theorem, we complete the proof.

Remark 6.1. The argument of the previous corollary may be applied to a more general case. Let $(Y, Q)$ be a random walk, and let $(\mathbb{Z}, P)$ be as in Theorem 6.1. Consider $Y \times \mathbb{Z}$ with transition matrix $\alpha\left(\mathbb{I}^{Y} \times P\right)+(1-\alpha)\left(Q \times \mathbb{I}^{\mathbb{Z}}\right)$, where $\alpha \in(0,1)$ and $\mathbb{I}$ denotes the identity matrix (on the superscripted space). Using the projection on the second coordinate, we prove that $\lim _{m \rightarrow+\infty} \lambda_{\mathrm{w}}^{m}=1=\lambda_{\mathrm{w}}$.

Theorem 6.2. If $(X, P)$ is the simple random walk on the homogeneous tree of degree $r$ then $\lim _{m \rightarrow+\infty} \lambda_{\mathrm{w}}^{m}=1=\lambda_{\mathrm{w}}$. 
Proof. Fix an end $\tau$ in $X$ and a root $o \in X$, and define the map $h: X \rightarrow \mathbb{Z}$ as the usual height (see [25, p. 129]). Define $A_{k}=h^{-1}(k), k \in \mathbb{Z}$ (these sets are usually referred to as horocycles). The projection of the simple random walk on $X$ onto $\mathbb{Z}$ is a random walk with transition matrix $\widetilde{P}$, where $\widetilde{p}(a, a+1)=1-1 / r$ and $\widetilde{p}(a, a-1)=1 / r$. Note that, for all $x \in X$,

$$
\sum_{y \in A_{k}} p^{(n)}(x, y)=\tilde{p}^{(n)}(h(x), k) .
$$

By using (6.1) and reasoning as in Lemma 5.3 and Theorem 6.1, we find that, for all $i \in \mathbb{N}$, and some integers $d_{1}$ and $d_{2}$,

$$
\mathrm{P}\left(\sum_{x \in A_{i+j}} \bar{\eta}_{\bar{n}}(x) \geq k, j=d_{1}, d_{2} \mid \bar{\eta}_{0}(x)=\eta\right)>1-\varepsilon
$$

for all $\eta$ such that $\sum_{x \in A_{i}} \eta(x)=k$ and $\eta(x)=0$ if $x \notin A_{i}$, and all sufficiently large $n_{0}$. The claim follows as in Theorem 6.1.

\section{BRWs in random environments}

We use the results of Section 3 to prove some properties of the BRW in a random environment.

Let $(X, \mu)$ be a nonoriented weighted graph. We consider any subgraph $(Y, \mathcal{E}(Y))$ of $(X, \mathcal{E}(X))$ as a weighted subgraph with weight function $\mathbf{1}_{\mathscr{E}(Y)} \mu$.

Given any $p \in(0,1]$, we consider the independent Bernoulli bond percolation on $(X, \mathcal{E}(X))$ and we define the random weighted subgraphs $\left(Y^{a}, \mathcal{E}\left(Y^{a}\right)\right)$, where $Y^{a}=X$ and $\mathscr{E}\left(Y^{a}\right)$ is the random set of edges resulting from the percolation process. We define $\lambda_{\mathrm{s}}\left(Y^{a}\right):=\inf _{A \in \mathcal{A}} \lambda_{\mathrm{s}}(A)$, where $\mathcal{A}$ is the random collection of all the connected components of $Y^{a}$. This corresponds to the critical (strong) parameter of a BRW where the initial state is one particle alive at time 0 in every connected component of $Y^{a}$.

On the other hand, if there exists a nontrivial critical parameter $p_{c}$ for the Bernoulli percolation on $X$ and if the infinite cluster is unique almost surely (a.s.) then, for all $p>p_{c}$, we denote by $\left(Y^{c}, \mathcal{E}\left(Y^{c}\right)\right)$ the infinite cluster and we consider the critical (strong) parameter $\lambda_{\mathrm{s}}\left(Y^{c}\right)$. Given a sequence $\left\{p_{n}\right\}_{n \in \mathbb{N}}$ such that $p_{n} \in[0,1]$ for all $n \in \mathbb{N}$, we consider the sequences $\left\{Y_{n}^{a}\right\}_{n \in \mathbb{N}}$ and $\left\{Y_{n}^{c}\right\}_{n \in \mathbb{N}}$ as the results of Bernoulli percolation processes on $X$ with parameters $\left\{p_{n}\right\}_{n \in \mathbb{N}}$. Observe that we do not make any a priori assumption on the joint distributions of these Bernoulli percolation processes.

Here is the main result; we note that, even when $X=\mathbb{Z}^{d}$, we do not require $\mu$ to be the simple random walk.

Theorem 7.1. (i) If $\sum_{n}\left(1-p_{n}\right)<+\infty$ then $\lambda_{\mathrm{s}}\left(Y_{n}^{a}\right) \rightarrow \lambda_{\mathrm{s}}(X)$ a.s.

(ii) If $(X, \mu)$ is quasi-transitive then $\lambda_{\mathrm{s}}\left(Y^{a}\right)=\lambda_{\mathrm{s}}(X)$ a.s.

(iii) If $X=\mathbb{Z}^{d}$ and $\sum_{n}\left(1-p_{n}\right)<+\infty$, then $\lambda_{\mathrm{s}}\left(Y_{n}^{c}\right) \rightarrow \lambda_{\mathrm{s}}\left(\mathbb{Z}^{d}\right)$ a.s.

(iv) If $X=\mathbb{Z}^{d}$, $\mu$ is translation invariant, and $p>p_{c}$, then $\lambda_{\mathrm{s}}\left(Y^{c}\right)=\lambda_{\mathrm{s}}\left(\mathbb{Z}^{d}\right)$ a.s.

Proof. (i) Using the Borel-Cantelli lemma we find that any finite connected subgraph of $X$ is eventually contained in a (random) connected component of $Y_{n}^{a}$ a.s. (since $\sum_{n}\left(1-p_{n}^{k}\right)<+\infty$ for all $k \in \mathbb{N}$ ). Theorem 3.1 yields the conclusion. 
(ii) In this case if we take an infinite orbit $X_{0}$ then, for any $m \in \mathbb{N}$, with probability $1, Y^{a}$ contains at least one ball $B\left(z_{0}, m\right)$, centered on a vertex $z_{0} \in X_{0}$ and of radius $m$, with all open edges. Indeed, for each $m$, there exists an infinite subset of $X_{0}$, say $X_{1}$, such that the balls $\left\{B(z, m): z \in X_{1}\right\}$ are pairwise disjoint. Hence, the events 'all the edges in $B(z, m)$ are open' with $z \in X_{1}$ are independent and they have the same probability; thus, a.s. (with respect to the percolation probability), at least one occurs. Let $B\left(z_{0}, m\right)$ be the corresponding ball ( $z_{0}$ being random).

Since the critical parameter of a ball $\lambda_{\mathrm{S}}\left(B\left(z_{0}, m\right)\right)$ does not depend on the choice of $z_{0} \in X_{0}$, then using Theorem 3.1, we have $\lambda_{\mathrm{s}}(X) \leq \lambda_{\mathrm{s}}\left(Y^{a}\right) \leq \lambda_{\mathrm{s}}\left(B\left(z_{0}, m\right)\right) \rightarrow \lambda_{\mathrm{s}}(X)$ as $m \rightarrow+\infty$.

(iii) Note that $p_{n}>p_{c}$ eventually; hence, $\lambda_{\mathrm{s}}\left(Y_{n}^{c}\right)$ is well defined for all sufficiently large $n$. What we need to prove is that, a.s., any edge is eventually connected to the infinite cluster. To this aim, we apply the FKG inequality, finding that the probability of the event 'the edge $(x, y)$ is open and connected to the infinite cluster $Y_{n}^{c}$, is bigger than $p_{n} \theta\left(p_{n}\right)$ (where $\theta(p)$ is the probability that a fixed vertex $x$ is contained in the infinite cluster, when each edge is open with probability $p$ ). According to Theorem 8.92 of [9], $\theta$ is a differentiable function on [0, 1]; hence, $1-p \theta(p) \sim(1-p)\left(1+\theta^{\prime}(1)\right)$, and this implies that $\sum_{n}\left(1-p_{n} \theta\left(p_{n}\right)\right)<+\infty$. The Borel-Cantelli lemma yields the conclusion.

(iv) It is tedious but essentially straightforward to prove that, for any $m \in \mathbb{N}$, with probability 1 , $Y^{c}$ contains a hypercube $Q_{m}$ of side length $m$ with all open edges; as before, Theorem 3.1 yields the result.

Note that, if $p_{n} \uparrow 1$ and the joint distribution of the family of Bernoulli percolation processes (indexed by $n$ ) is the natural monotone coupling, then the conclusions of Theorem 7.1(i) and (iii) hold without requiring that $\sum_{n}\left(1-p_{n}\right)<+\infty$. This stronger assumption nevertheless allows us to obtain a convergence result for all possible couplings.

\section{Final remarks}

At this point the theory of spatial approximation (see Section 3) is quite complete, as far as we are concerned with the basic questions regarding the convergence of the critical parameters. Indeed, we proved results in this direction (see Theorem 3.1) for the strong parameter under reasonable assumptions, while the question regarding the weak critical parameter, in the pure spatial approximation by finite subsets, is uninteresting (see Remark 3.1). It is possible to further investigate the convergence of the sequence of weak critical parameters under the hypotheses of Theorem 3.1 by using the characterization $\lambda_{\mathrm{w}}=1 / \lim \sup _{n} \sqrt[n]{\sum_{y \in X} \mu^{(n)}(x, y)}$, which holds in many cases (see [1] and [2] for details).

As for the approximation of the BRW by $\mathrm{BRW}_{m} \mathrm{~s}$, we proved that, on graphs satisfying the hypotheses of Theorem 5.1 (among them, quasi-transitive graphs), $\lambda_{\mathrm{s}}^{m} \downarrow \lambda_{\mathrm{s}}$ as $m \rightarrow \infty$ and, if there is no weak phase, on $\mathbb{Z}^{d}$ or on regular trees, $\lambda_{\mathrm{w}}^{m} \downarrow \lambda_{\mathrm{w}}$ as $m \rightarrow \infty$. Here are some natural questions which, as far as the authors are aware, are still open.

- Can we weaken the hypothesis of Theorem 5.1 and still prove the convergence of the strong critical parameters?

- When $\lambda_{\mathrm{w}} \neq \lambda_{\mathrm{s}}$, is it still true that $\lambda_{\mathrm{w}}^{m} \downarrow \lambda_{\mathrm{w}}$ as $m \rightarrow \infty$, at least for Cayley graphs or on quasi-transitive graphs? 


\section{Acknowledgements}

The authors are grateful to Rick Durrett for his invaluable suggestions. They would also like to thank an anonymous referee for his/her comments which helped to clarify some proofs.

\section{References}

[1] Bertacchi, D. ANd ZucCA, F. (2008). Critical behaviors and critical values of branching random walks on multigraphs. J. Appl. Prob. 45, 481-497.

[2] Bertacchi, D. AND ZuCCA, F. (2009). Characterization of the critical values of branching random walks on weighted graphs through infinite-type branching processes. J. Statist. Phys. 134, 53-65

[3] Bertacchi, D., Posta, G. and Zucca, F. (2007). Ecological equilibrium for restrained random walks. Ann. Appl. Prob. 17, 1117-1137.

[4] Bramson, M. and Durrett, R. (1988). A simple proof of the stability criterion of Gray and Griffeath. Prob. Theory Relat. Fields 80, 293-298.

[5] Coulhon, T., Grigor'yan, A. And Zucca, F. (2005). The discrete integral maximum principle and its applications. Tohoku Math. J. 57, 559-587.

[6] Durrett, R. (1995). Ten Lectures on Particle Systems (Lectures Notes Math. 1608). Springer, Berlin.

[7] Durrett, R. and Neuhauser, C. (1991). Epidemics with recovery in D=2. Ann. Appl. Prob. 1, 189-206.

[8] Ethier, S. N. And Kurtz, T. G. (1986). Markov Processes. John Wiley, New York.

[9] Grimmett, G. (1999). Percolation. Springer, Berlin.

[10] Harris, T. E. (1960). A lower bound for the critical probability in a certain percolation process. Proc. Camb. Phil. Soc. 56, 13-20.

[11] Hueter, I. ANd Lalley, S. P. (2000). Anisotropic branching random walks on homogeneous trees. Prob. Theory Relat. Fields 116, 57-88.

[12] Liggett, T. M. (1996). Branching random walks and contact processes on homogeneous trees. Prob. Theory Relat. Fields 106, 495-519.

[13] LiggetT, T. M. (1999). Branching random walks on finite trees. In Perplexing Problems in Probability (Progress Prob. 44), Birkhäuser, Boston, MA, pp. 315-330.

[14] Liggett, T. M. And SpItZER, F. (1981). Ergodic theorems for coupled random walks and other systems with locally interacting components. Z. Wahrscheinlichkeitsth. 56, 443-468.

[15] Lyons, R. (2000). Phase transitions on nonamenable graphs. Probabilistic techniques in equilibrium and nonequilibrium statistical physics. J. Math. Phys. 41, 1099-1126.

[16] Madras, N. And Schinazi, R. (1992). Branching random walks on trees. Stoch. Process. Appl. 42, $255-267$.

[17] Mountford, T. And Schinazi, R. (2005). A note on branching random walks on finite sets. J. Appl. Prob. 42, 287-294.

[18] Neuhauser, C. (1992). Ergodic theorems for the multitype contact process. Prob. Theory Relat. Fields 91, 467-506.

[19] Pemantle, R. and Stacey, A. M. (2001). The branching random walk and contact process on Galton-Watson and nonhomogeneous trees. Ann. Prob. 29, 1563-1590.

[20] Seneta, E. (2006). Non-Negative Matrices and Markov Chains. Springer, New York.

[21] Schinazi, R. (2003). On the role of social clusters in the transmission of infectious diseases. J. Theoret. Biol. 225, 59-63.

[22] Schinazi, R. (2005). Mass extinctions: an alternative to the Allee effects. Ann. Appl. Prob. 15, 984-991.

[23] Stacey, A. M. (2003). Branching random walks on quasi-transitive graphs. Combinatorics Prob. Comput. 12, 345-358.

[24] Van Den Berg, J., Grimmett, G. R. and Schinazi, R. B. (1998). Dependent random graphs and spatial epidemics. Ann. Appl. Prob. 8, 317-336.

[25] Woess, W. (2000). Random Walks on Infinite Graphs and Groups (Camb. Tracts Math. 138). Cambridge University Press. 